\title{
DC Conductivity and Spectroscopic Characterization of Poly (o-toluidine) Doped with Binary Dopant $\mathrm{ZrOCl}_{2} / \mathrm{AgI}$
}

\author{
Kiran Kumari ${ }^{1}$, Vazid Ali $^{1 *}$, Gita Rani ${ }^{1}$, Sushil Kumar ${ }^{2}$, G. B. V. S. Lakshmi ${ }^{3}$, M. Zulfequar ${ }^{3}$ \\ ${ }^{1}$ Advanced Materials Research Lab, Department of Chemistry, Chaudhary Devi Lal University, Sirsa, India; ${ }^{2}$ Department of Physics, \\ Chaudhary Devi Lal University, Sirsa, India; ${ }^{3}$ Materials Science Lab, Department of Physics, Jamia Millia Islamia, (Central Univer- \\ sity), New Delhi, India. \\ Email: poly3resmat@gmail.com
}

Received November $25^{\text {th }}, 2010$; revised January $19^{\text {th }}, 2011$; accepted June $10^{\text {th }}, 2011$.

\begin{abstract}
Aqueous binary dopant $\left(\mathrm{ZrOCl}_{2} / \mathrm{AgI}\right)$ is used in different ratio such as 1:1, 1:2 and 2:1 (w/w) for chemical doping to enhance the conductivity of synthesized Poly (o-toluidine) (POT). The doping of Poly (o-toluidine) is carried out using tetrahydrofuran as solvent. Doped samples are characterized using various techniques such as I-V characteristics, UV-Visible spectroscopy, X-ray diffractometry (XRD), FTIR and Photoluminescence (PL) studies. A significant enhancement in DC conductivity has been observed with the introduction of binary dopant. UV-Visible study shows that optical parameters change considerably after doping. Interestingly, both direct and indirect band gaps are observed in the doped samples. XRD patterns show the semi-crystalline nature of doped Poly (o-toluidine). FTIR study shows structural modifications in functional groups with doping in POT. A Photolyminescence spectrum exhibits the emission properties of the samples.
\end{abstract}

Keywords: Poly (O-Toluidine), Dc Conductivity, UV-Visible Study, X-Ray Diffraction, FT-IR and Photoluminescence Studies

\section{Introduction}

Polymers are typically utilized in electrical, optical and electronic devices as insulators because of their very high electrical resistivity. The dielectric properties of heterogeneous polymers [1] play an important role in device applications such as high performance capacitors, electrical cable insulation, electronic packaging etc. Polymers are usually polyconjugated structures, which are insulators in their pure state; but when treated with oxidizing or reducing agents they can be converted into polymer salts having reasonable electrical conductivity. Conjugated polymers are plastic semiconductors [2]. They have wide applications in devices such as solar cells, rechargeable batteries, light emitting diodes, micro-actuators, electrochromic displays, field effect transistors, sensors [3].

In polymers, different processes such as can carry out doping chemical and electrochemical. Recently metal salt doping in Poly (o-toluidine) has been reported [4]. Among these polymers, Poly (o-toluidine) has attracted much attention of many researchers due to its ease of synthesis, processibility, good thermal stability and good environmental stability. MacDiarmid et al. [5] investigated Poly (o-toluidine) as an electrically conducting polymer, which is emerging as a promising synthetic metal. The possibility of synthesizing and doping of Poly (o-toluidine) with protonic acid dopants containing different types of counterions is one of the key factors responsible for the versatility of this class of polymers. Photoluminescent organic molecules are a new class of compounds with interesting properties. They undergo emission over a wide range from the violet to the red. They can also be combined in several different forms to produce white light. One category of organic material with photoluminescence properties is conjugated organic polymers.

In the present work, our approach is to study the binary metal salt $\left(\mathrm{ZrOCl}_{2} / \mathrm{AgI}\right)$ induced chemical doping in Poly (o-toluidine) in the presence of distilled water and tetrahydrofuran. The reason for choosing the combina- 
tion of $\mathrm{ZrOCl}_{2}$ and $\mathrm{AgI}$ as binary dopant is that $\mathrm{ZrOCl}_{2}$ is a luminescent material contributing towards photoluminescence; while AgI is a conducting material and hence contributing towards dc conductivity. Therefore, by choosing such combination, we can prepare a material having good conductivity as well as phololuminescence simultaneously. Sincere efforts have been made to understand the effect of binary dopant on the electrical and spectroscopic properties of Poly (o-toluidine) using I-V characteristics method, UV-visible spectroscopy, X-ray diffractometry (XRD), FTIR and Photoluminescence (PL) studies.

\section{Experimental Details}

\subsection{Chemicals}

o-toluidine (Loba Chemie Pvt. Ltd. Mumbai, 99\%), Potassium dichromate (S.D. Fine Chemicals Ltd. Bombay A.R. grade), Hydrochloric acid (Qualigens fine Chemicals Ltd. Bombay A.R. Grade), Ammonia solution in water 28\% (S.D. Fine Chemicals Ltd. Bombay), Tetrahydrofuran (Merck India Ltd. Bombay A.R. Grade), $\mathrm{ZrOCl}_{2}$ (S.D. Fine Chemical, A.R. Grade) and AgI (HiMedia Lab., A.R. Grade). The binary dopant $\left(\mathrm{ZrOCl}_{2} / \mathrm{AgI}\right)$ is prepared by mixing their powders homogenously taken as per their $(\mathrm{w} / \mathrm{w})$ ratio $(1: 1,1: 2,2: 1)$.

\subsection{Synthesis and Chemical Doping}

Distilled o-toluidine is used to synthesize Poly (o-toluidine) (POT) by chemical oxidation polymerization in acidic medium as suggested by Mac Diarmid [6]. Synthesized Poly (o-toluidine) (POT) is dried in oven and grinded to obtain POT powder. Three system of binary dopant $\left(\mathrm{ZrOCl}_{2} / \mathrm{AgI}\right)$ having ratio $(1: 1,1: 2$ and $2: 1)$ $(\mathrm{w} / \mathrm{w})$ are used. $2.0 \mathrm{gm}$ POT powder and dopant having $2 \%(\mathrm{w} / \mathrm{w})$ concentration has been used in $10 \mathrm{ml}$ tetrahydrofuran (THF) solvent with magnetic stirring about 15 min, and then kept it in oven at $30^{\circ} \mathrm{C}$ for $24 \mathrm{~h}$ to perform doping process completely in Poly (o-toluidine). The physical state of binary dopant $\mathrm{ZrOCl}_{2} / \mathrm{AgI}$ is solid particles in suspension appeared milky in tetrahydrofuran (THF). After this chemically doped Poly (o-toluidine) was put in oven at $110^{\circ} \mathrm{C}$ for $4 \mathrm{~h}$ to achieve moisture free doped Poly (o-toluidine).

\section{Results and Discussion}

\subsection{Dc Conductivity}

DC conductivity of undoped and $\left(\mathrm{ZrOCl}_{2} / \mathrm{AgI}\right)$ doped Poly (o-toluidine) of the pellets (1.0 mm thickness) was measured by using two-probe method at temperature (298K). Dc conductivity was measured by mounting them between steel electrodes inside a specially designed sample holder. The temperature was measured with a calibrated copper-constantan thermocouple mounted near the electrodes. The samples were annealed to avoid any effect of moisture absorption. These measurements were made at a pressure of about $10^{-3}$ Torr. A stabilized voltage of $1.5 \mathrm{~V}$ was applied across the sample and the resultant current was measured with a pico- ammeter, which gives De conductivity within $\pm 1 \%$ of accuracy [7]. Conductivity is measured by using Ohm's law,

$$
\mathrm{V}=\mathrm{RI}
$$

where, $\mathrm{I}$ is the current (in Amperes) through a resistor $\mathrm{R}$ (in ohms) and $\mathrm{V}$ is the drop in potential (in volts) across it. The reciprocal of resistance $\left(\mathrm{R}^{-1}\right)$ is called conductance, the flow of current I as a result of gradient in potential leads to energy being dissipated $\left(\mathrm{RI}^{2}\right.$ joule $\left.\mathrm{s}^{-1}\right)$.

In Ohmic material, the resistivity measured is proportional to the sample cross-section A and inversely propotional to its length 1 :

$$
\mathrm{R}=\rho \mathrm{l} / \mathrm{A}
$$

where $\rho$ is the resistivity measured in $\Omega \mathrm{cm}$. Its inverse $\sigma$ $=\rho^{-1}$ is the conductivity. It is found that Dc conductivity of $\left(\mathrm{ZrOCl}_{2} / \mathrm{AgI}\right)$ doped POT samples changes with changes their ratio, and is shown in Table 1.

Dc-conductivity of pure POT increases exponentially with temperature, exhibiting semi conductor behaviour.

The doping of conducting polymers implies charge transfer, the associated insertion of a counter ion and the simultaneous control of Fermi level or chemical potential. The electrical conductivity of conducting polymers results from mobile charge carriers introduced into $\pi$ - electronic system through doping. At low doping levels these charge carriers are self-localized and form non-linear configuration. Because of large interchain transfer integrals, the transport of charge is believed to be principally along the conjugated chains, with interchain hopping as a necessary secondary condition [8]. In POT, there are nearly degenerate ground states, the dominating charge carriers are polarons and bipolarons. It has been observed that $\mathrm{ZrOCl}_{2} / \mathrm{AgI}$ doped POT is showing charge carriers formation with linear configuration; as a result, conductivity changes substantially.

\subsection{Optical Studies}

UV-Visible study of doped Poly (o-toluidine) samples is performed using Perkin Elmer Lambda Spectrophotometer. Optical parameters such as absorption coefficient $(\alpha)$, molar absorptivity or extinction coefficient $(k)$ and energy band gap (Eg) have been determined for undoped and $\left(\mathrm{ZrOCl}_{2} / \mathrm{AgI}\right)$ ratio $(1: 1,1: 2,2: 1)$ doped Poly (o-toluidine) samples at $298 \mathrm{~K}$ through absorption spectra. The relationship between the optical band gap (Eg), absorption coefficient $(\alpha)$ and the photon energy (hv) of the incident photon is given by [9-10]; 
Table 1. Optical parameters and DC-conductivity of Poly (o-toluidine) doped with $\left(\mathrm{ZrOCl}_{2} / \mathrm{AgI}\right)$ doping system ratio $(1: 1,1: 2$ and $2: 1(w / w))$ at $2 \%$ dopant concentration.

\begin{tabular}{|c|c|c|c|c|c|c|}
\hline $\begin{array}{c}\text { Ratio of } \\
\left(\mathrm{ZrOCl}_{2} / \mathbf{A g I}\right) \\
(\mathrm{w} / \mathrm{w})\end{array}$ & $\begin{array}{c}\text { Dopant } \\
\text { (ZrOCl } 2 / \text { AgI) } \\
\text { Concentration in } \\
\text { Poly (o-toluidine) } \\
\text { (w/w) }\end{array}$ & $\begin{array}{l}\text { Direct band } \\
\text { gap Eg }(\mathrm{eV})\end{array}$ & $\begin{array}{c}\text { Indirect band } \\
\text { gap Eg }(\mathrm{eV})\end{array}$ & $\begin{array}{c}\text { Absorption } \\
\text { coefficient } \alpha \\
\text { at } \lambda=400 \mathbf{~ n m}\end{array}$ & $\begin{array}{c}\text { Extinction } \\
\text { coefficient } \mathbf{k} \\
\text { at } \lambda=400 \mathbf{n m}\end{array}$ & $\begin{array}{c}\text { Conductivity } \sigma \\
(\mathrm{S} / \mathrm{cm})\end{array}$ \\
\hline & Undoped & 2.75 & 1.83 & 0.99 & 31.82 & $0.67 \times 10^{-8}$ \\
\hline $1: 1$ & $2 \%$ doped & 1.44 & 1.45 & 1.50 & 47.73 & $0.73 \times 10^{-5}$ \\
\hline $1: 2$ & $2 \%$ doped & 1.36 & 1.41 & 1.40 & 44.55 & $0.68 \times 10^{-5}$ \\
\hline $2: 1$ & $2 \%$ doped & 1.38 & 1.39 & 0.90 & 28.64 & $0.78 \times 10^{-5}$ \\
\hline
\end{tabular}

$$
\alpha \mathrm{h} v \propto(\mathrm{h} v-\mathrm{Eg})^{\mathrm{n}}
$$

where $n=1 / 2,3 / 2, \ldots$ for direct and $n=1,2,3, \ldots \ldots$ for indirect transitions respectively.

It has been observed that both undoped and doped Poly (o-toluidine) samples, show direct and indirect transitions. The direct and indirect band gaps both show decreasing orders; The extinction coefficient $(k)$ is determined by the following relation,

$$
k=\alpha \lambda / 4 \pi
$$

Absorption coefficient and extinction coefficient have been determined using sharp increase of UV- Visible absorption spectra at wavelength $400 \mathrm{~nm}$ as shown in Figure 1. There are rising bends/curves at wavelength $380 \mathrm{~nm}$, which is attributed to oxidized phase of the Poly (o-toluidine), another moderate peaks at $\sim 400 \mathrm{~nm}$ appears for all studied samples. In each ratio of doping, $2 \%$ (w/w) doped sample attributed to transition with significant shifting of peak at $400 \mathrm{~nm}$, which is also further showing the changes in optical and electrical properties (Table 1).

It has also been observed, in $\left(\mathrm{ZrOCl}_{2} / \mathrm{AgI}\right)$ doped Poly (o-toluidine) sample, that there is change in band gap $(\mathrm{Eg})$ at different ratio $(1: 1,1: 2$ and $2: 1)(\mathrm{w} / \mathrm{w})$ the concentration of dopant increase. Absorption coefficient and extinction coefficient changes with changing ratio.

Conclusively, $\left(\mathrm{ZrOCl}_{2} / \mathrm{AgI}\right)$ play a significant role in the chemical doping of Poly (o-toluidine), the measured optical parameters is showing the significant changes. Thus, the information about the changes in optical parameters by chemical doping with $\left(\mathrm{ZrOCl}_{2} / \mathrm{AgI}\right)$ may explore the possibilities in the course of development with new metal salts systems in conducting polymers.

\subsection{XRD Studies}

XRD patterns provide information in relation to the nature and structure of the samples. XRD pattern of undoped sample of Poly (o-toluidine) shows the amorphous nature. XRD patterns of doped samples (increasing carrier's concentration) show the semi-crystallinity. The
DC-conductivity of samples increases due to the increase in crystallinity of the sample.

Since the conductivity of polymers depends on various parameters such as doping level (carrier's concentration), formation of polarons and bipolarons [11], the semicrystalline nature of polymers arises owing to the systematic alignment of polymer chain folding or by the formation of single or multiple helices, for part of their length [12]. XRD patterns of undoped and doped poly (o-toluidine) with $2 \%$ doping concentration and binary dopant ratio $1: 1,1: 2,2: 1$ are shown in Figure 2(a)-(d). XRD pattern of undoped POT sample shows an amorphous hump around $28^{\circ}$ and the doped samples have peak at $4^{\circ}$. As shown in Figure 2(b), broadening of peaks in XRD pattern have been obtained at about $27^{\circ}-28^{\circ}$ corresponding to ratio 1:2 binary dopant. In 2:1 doped sample, there are three small peaks at $21^{\circ}, 23^{\circ}$ and $39^{\circ}$. The variation in diffraction intensity with dopant concentration exhibits with the interaction of dopant in POT network.

\subsection{FTIR Studies}

FTIR Spectra have been recorded for undoped and doped POT $\left(2 \%\right.$ doped) with binary dopant $\left(\mathrm{ZrOCl}_{2} / \mathrm{AgI}\right)$ ratio 1:1, $1: 2$ and $2: 1(\mathrm{w} / \mathrm{w})$ are shown in Figure 3(a)-(d). Spectra have been recorded for undoped and doped POT with binary dopant $\left(\mathrm{ZrOCl}_{2} / \mathrm{AgI}\right)(\mathrm{w} / \mathrm{w})$ ratio $1: 1,1: 2$ and 2:1 are shown in Figure 3(a)-(d). The broad medium band at $3421 \mathrm{~cm}^{-1}$ in 1:1 $\left(\mathrm{ZrOCl}_{2} / \mathrm{AgI}\right)(\mathrm{w} / \mathrm{w})$ doped and at $3439 \mathrm{~cm}^{-1}$ in $2: 1\left(\mathrm{ZrOCl}_{2} / \mathrm{AgI}\right)(\mathrm{w} / \mathrm{w})$ doped POT have been observed. These vibrational bands observed may be explained based on the normal modes of Poly (o-toluidine). The medium intensity band at $1586 \mathrm{~cm}^{-1}$ (as in undoped POT) is assigned to the C-N stretching of secondary aromatic amine, which shifts to $1616 \mathrm{~cm}^{-1}$ in 2:1 $\left(\mathrm{ZrOCl}_{2} / \mathrm{AgI}\right)(\mathrm{v} / \mathrm{v})$ doped samples. The band at $\sim 416$ $\mathrm{cm}^{-1}$ observed for undoped and doped POT samples is the characteristic peak of $\mathrm{C}-\mathrm{H}$ out of plane blending vibration of benzene ring [13-14]. On comparing the IR spectra of undoped and doped POT samples, the medium band observed (in doped samples) around $1587 \mathrm{~cm}^{-1}$ is 
the characteristic peak of nitrogen quinoid ring and is absent in Poly (o-toluidine) sample. The vibration band at $416 \mathrm{~cm}^{-1}$ is assigned to the benzene ring distribution, whose intensity increases with increase in dopant ratio $1: 1\left(\mathrm{ZrOCl}_{2} / \mathrm{AgI}\right)(\mathrm{w} / \mathrm{w})$. The changes in number and intensity of IR vibrational bands confirmed the dopant interaction with Poly (o-toluidine).

\subsection{Photoluminescence Studies}

The photoluminescence spectroscopy (PL) of

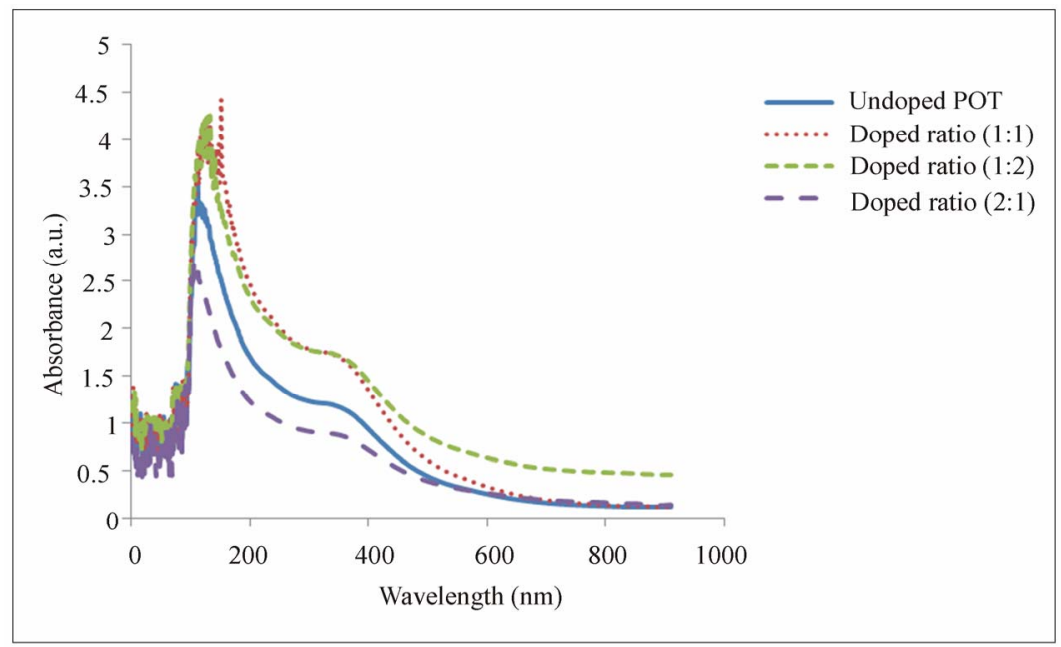

Figure1. Uv-Visible absorption spectra of undoped and doped Poly (o-toluidine) at different ratio of $1: 1\left(\mathrm{ZrOCl}_{2} / \mathrm{AgI}\right)(\mathrm{w} / \mathrm{w})$, 1:2 $\left(\mathrm{ZrOCl}_{2} / \mathrm{AgI}\right)(\mathrm{w} / \mathrm{w})$ and 2:1 $\left(\mathrm{ZrOCl}_{2} / \mathrm{AgI}\right)(\mathrm{w} / \mathrm{w})$ dopant mixture.

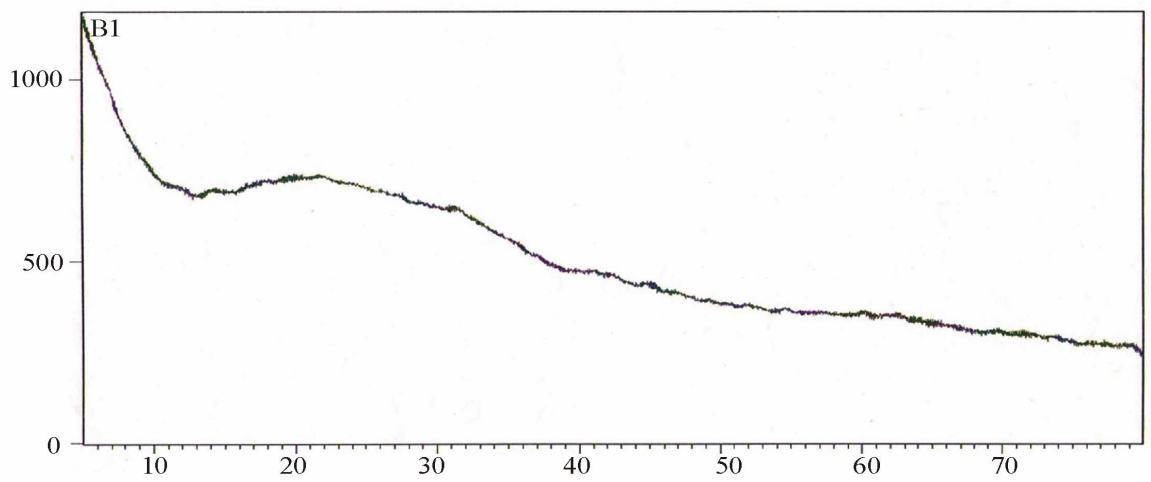

(a)

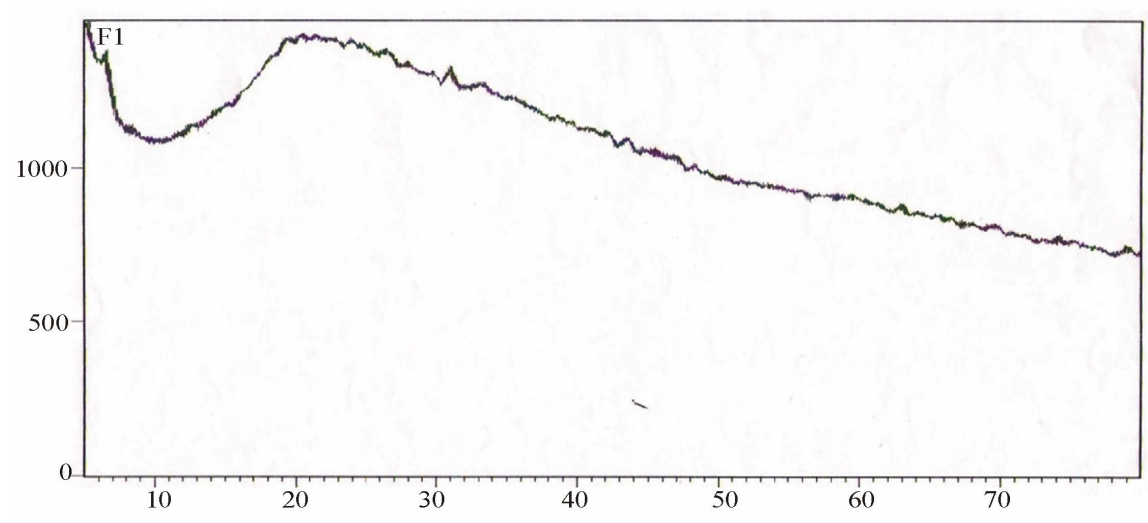

(b) 


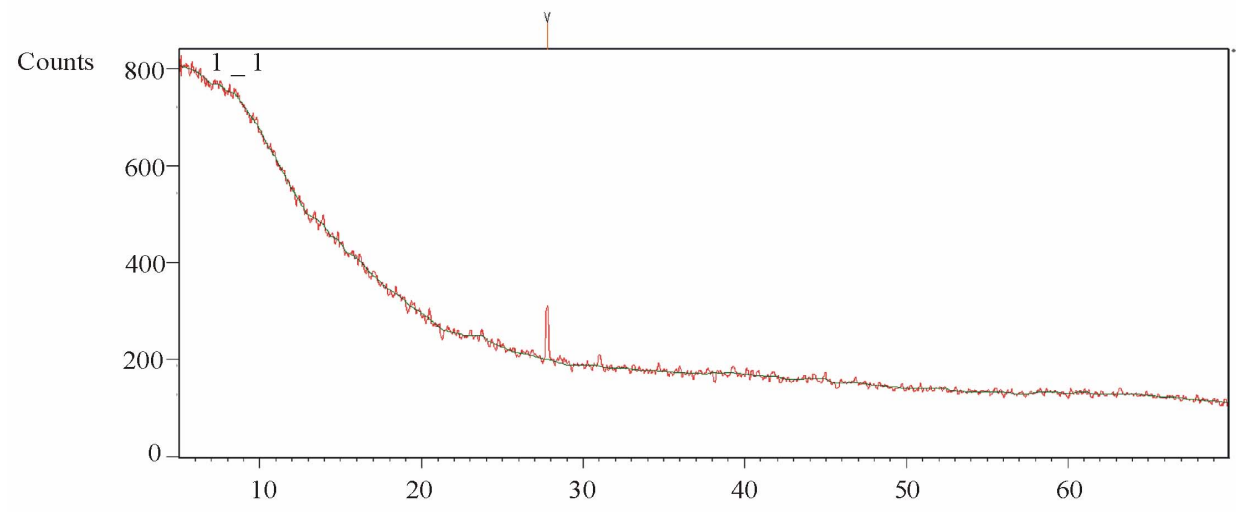

(c)

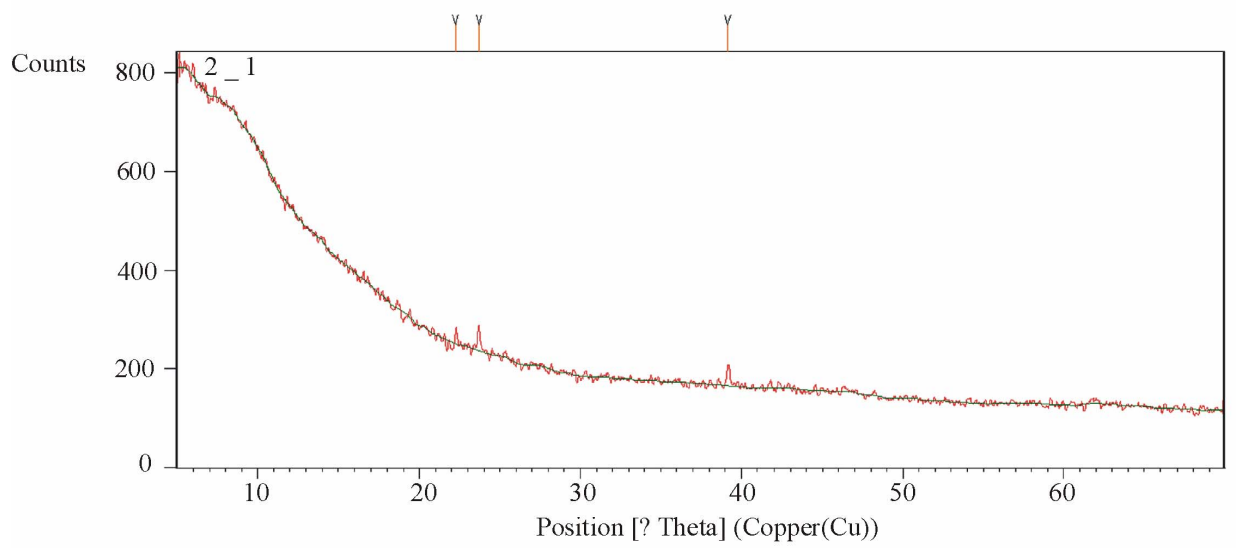

(d)

Figure 2. (a,b,c \& d): X-ray diffraction pattern of undoped and doped POT with ratio of $1: 1 \quad\left(\mathrm{ZrOCl}_{2} / \mathrm{AgI}^{\mathrm{O}}\right)(\mathrm{w} / \mathrm{w}), 1: 2$ $\left(\mathrm{ZrOCl}_{2} / \mathrm{AgI}\right)(\mathrm{w} / \mathrm{w})$ and 2:1 $\left(\mathrm{ZrOCl}_{2} / \mathrm{AgI}\right)(\mathrm{w} / \mathrm{w})$ dopant mixture.

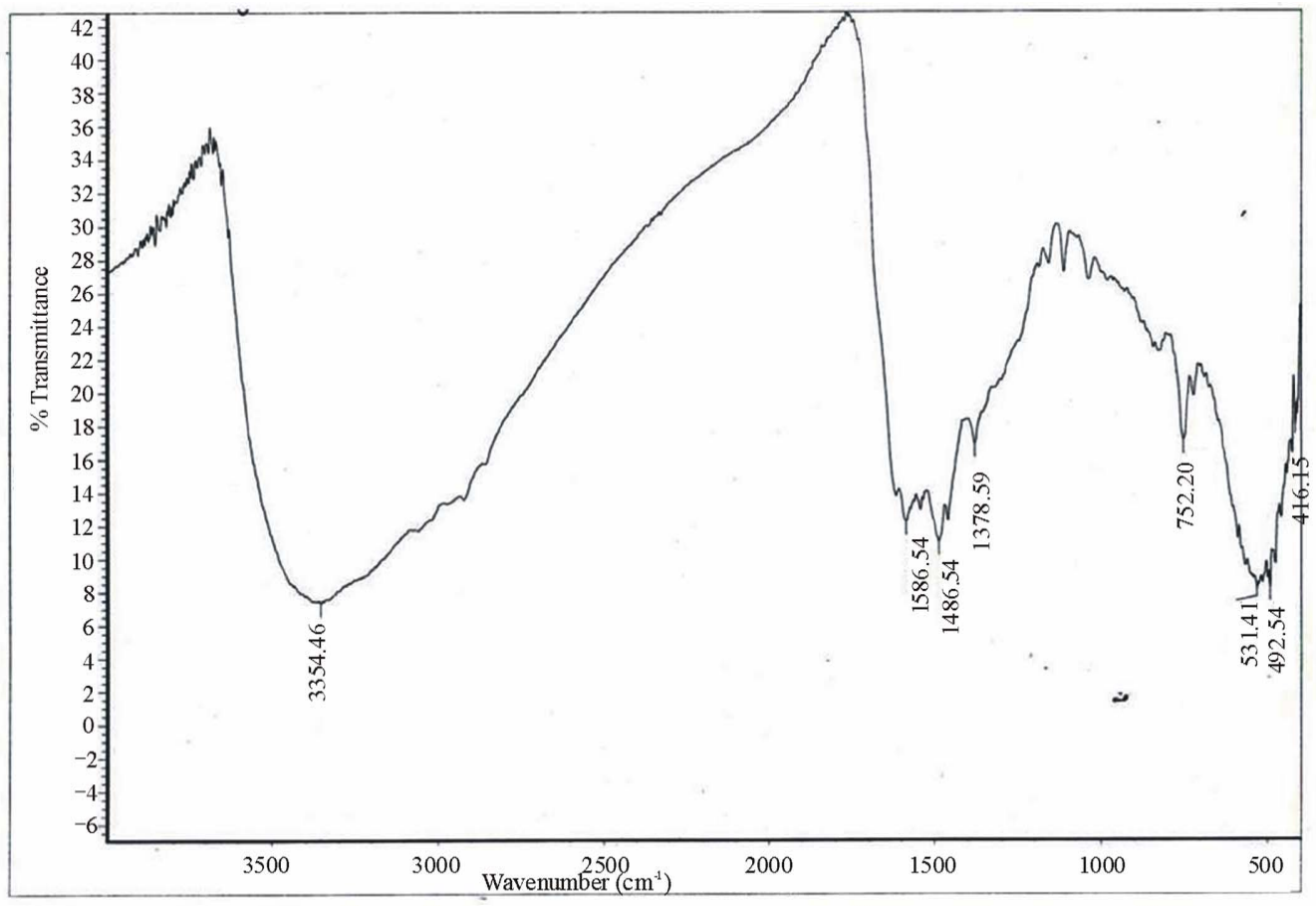

(a) 


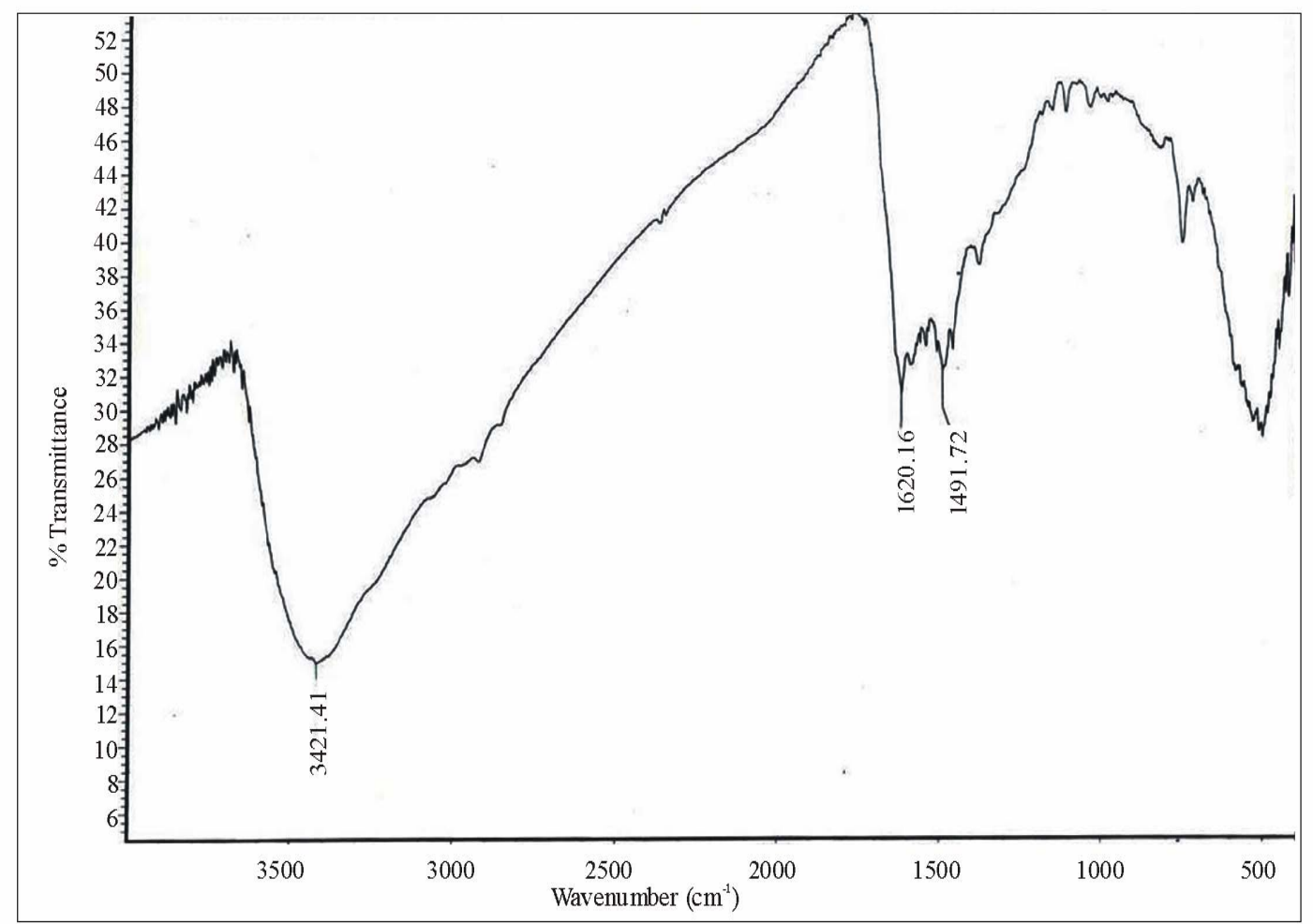

(b)

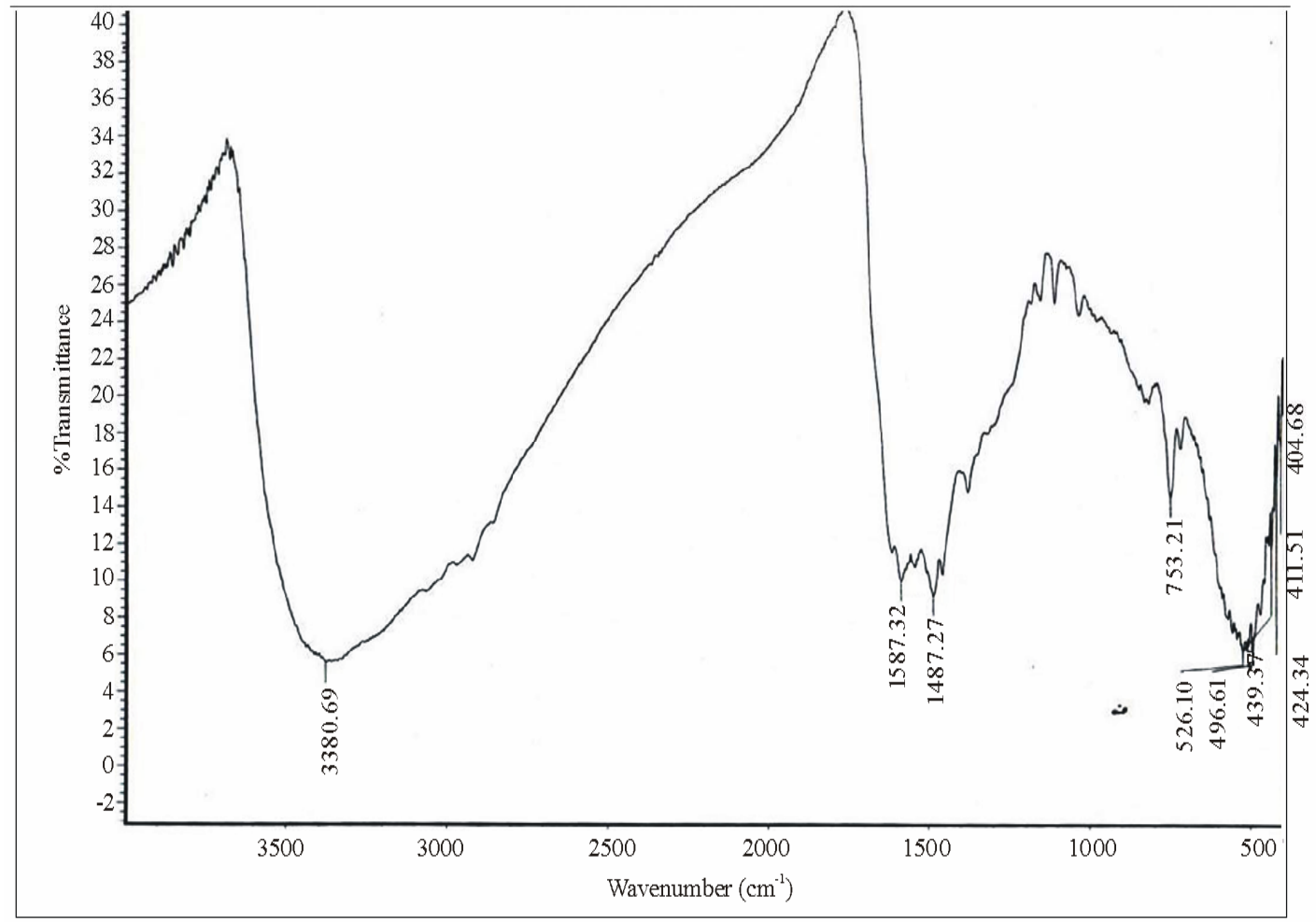

(c) 


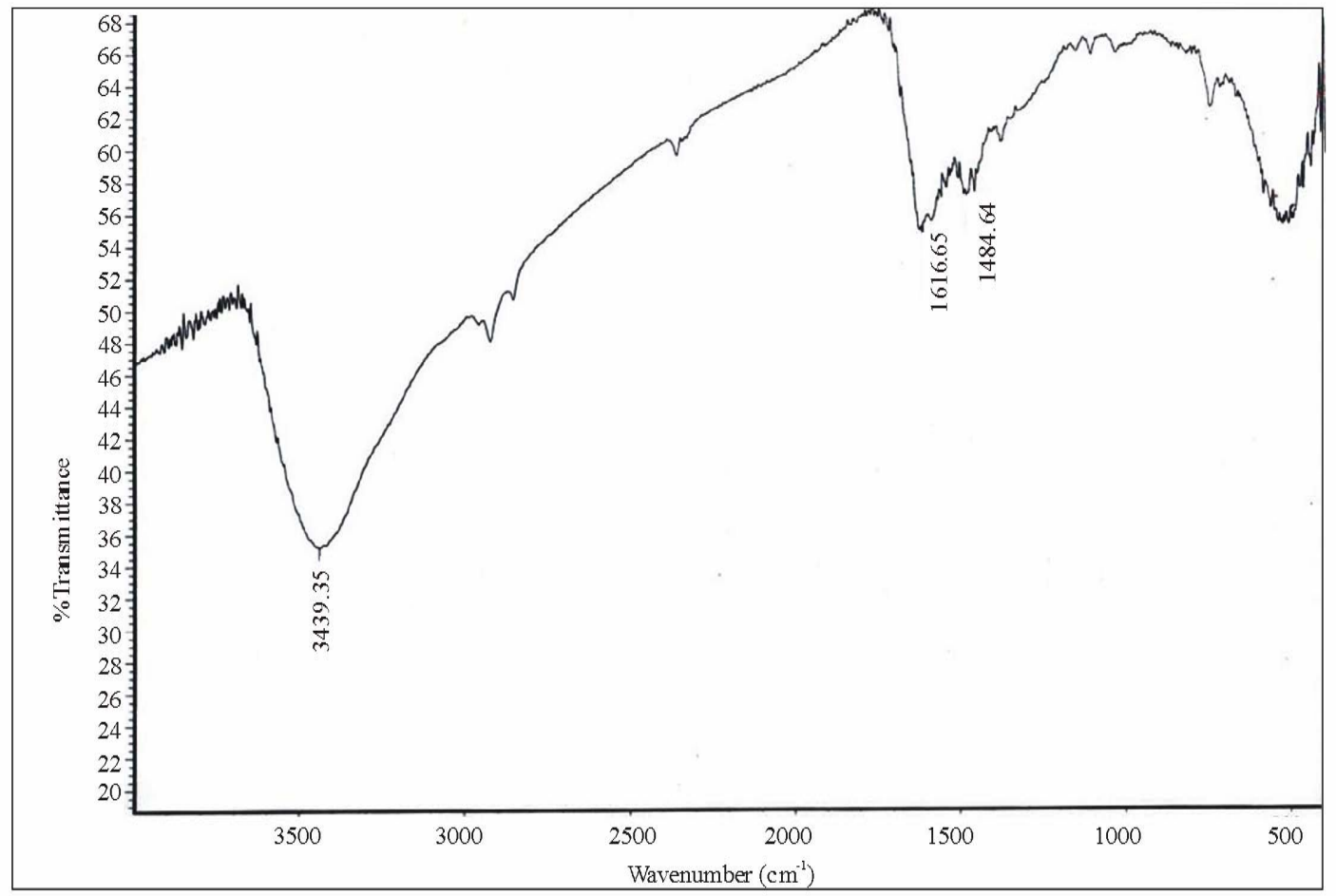

Figure 3. (a, b, c\&d):Photoluminescence spectra of undoped and doped POT with ratio of 1:1 ( $\left.\mathrm{ZrOCl}_{2} / \mathrm{AgI}\right)(\mathrm{w} / \mathrm{w}), 1: 2$ $\left(\mathrm{ZrOCl}_{2} / \mathrm{AgI}\right)(\mathrm{w} / \mathrm{w})$ and 2:1 $\left(\mathrm{ZrOCl}_{2} / \mathrm{AgI}\right)(\mathrm{w} / \mathrm{w})$ dopant mixture.

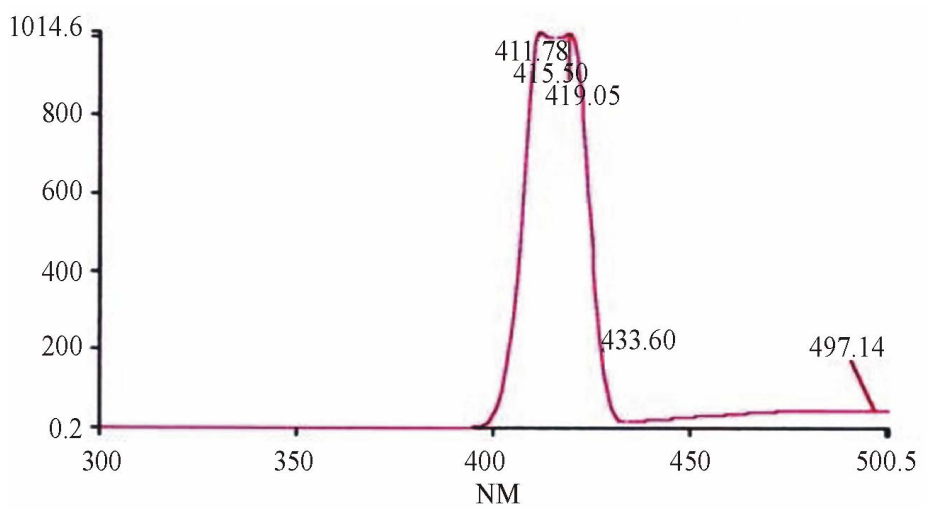

(a)

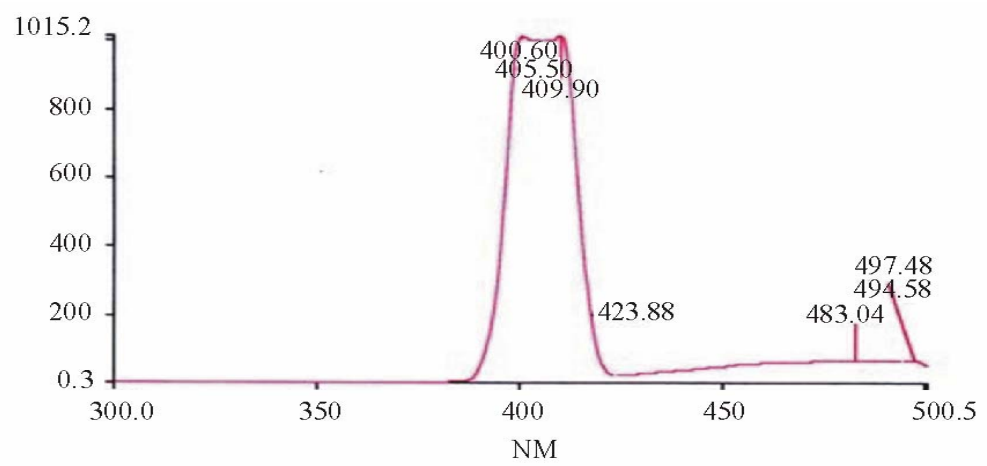

(b) 


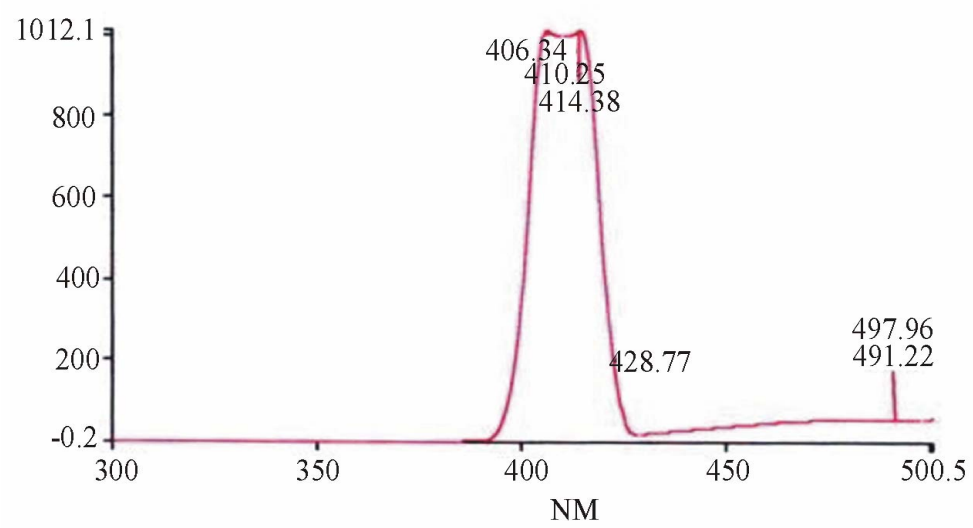

(c)

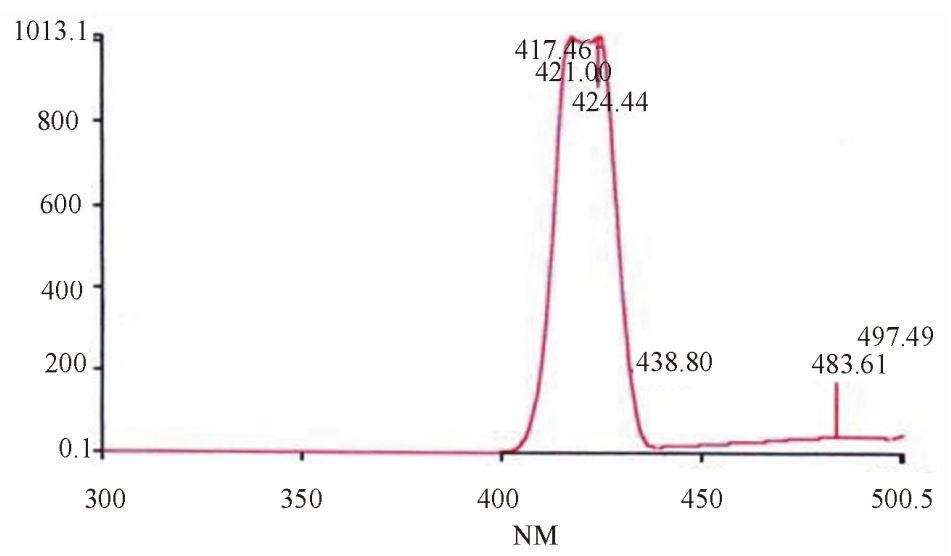

(d)

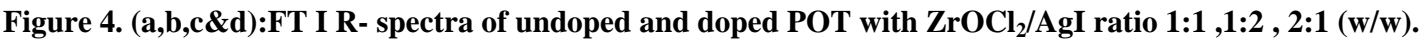

$\left(\mathrm{ZrOCl}_{2} / \mathrm{AgI}\right)(\mathrm{w} / \mathrm{w})$ doped POT has been performed and is shown in Figure 4 (a-d). It is found that the relative intensity of emission peaks alter with different ratio of binary dopant and nature of solvent (due to polarity). It has been noticed that the peak observed at $400 \mathrm{~nm}$ in undoped POT shifts towards higher wavelength with change in binary dopant ratio, i.e. 1:1, 1:2 and 2:1. In addition, this peak becomes sharp and intense in the sample having ratio $2: 1\left(\mathrm{ZrOCl}_{2} / \mathrm{AgI}\right)(\mathrm{w} / \mathrm{w})$ of binary dopant. This may be due to interchain species, which plays an important role in the emission process of conjugated polymers. The intensity of peaks depends on factors such as polymer coil size, the nature of polymer-solvent, polymer-dopant interactions, and the degree of chain overlapping [15]. The PL spectra of samples have the same shape, which indicates that it is an efficient way to tune the intensities of the peak by employing specific dopant with different compositions/ratio.

\section{Conclusions}

The present research work describes the strong influence of aqueous binary dopant $\left(\mathrm{ZrOCl}_{2} / \mathrm{AgI}\right)$ in Poly (o-toluidine) (o-toluidine) especially for optical and electrical properties. Doping induces a considerable change in optical properties of Poly (o-toluidine) such as optical band gap, absorption coefficient. It is interesting, that samples are showing both direct and indirect band gaps, which change with change in dopants ratio. Absorption coefficient and extinction coefficient also change with change in dopants ratio. Dc conductivity of doped samples is enhanced by three orders. XRD and FTIR spectra of doped POT indicate the strong interaction of dopant with POT $\pi$-conjugation system. The substantial structural modifications of doped POT occur as confirmed by XRD and FTIR spectra. The intensity the emission peaks alter with different dopant ratio and nature of solvents (due to polarity).

\section{Acknowledgements}

Authors wish to express their grateful thanks to Materials Science Laboratory, Department of Physics, Jamia Millia Islamia (Central University), New Delhi, and Punjab University, Chandigarh, India, for providing the experimental facilities. 


\section{REFERENCES}

[1] J. Planes, A. Wolter, Y. Cheguettine, A. Pron, F. Genobd and M. Nechtschein, "Polyaniline: Synthesis, Characterization, Solution Properties and Composites," Physical Reviews B, Vol. 58, 1998, pp. 7774-7778.

[2] R. H. Friend, R. W. Gymer and A. B. Holmes, "Electroluminescence in Conjugated Polymers," Nature, Vol. 397, 1997, pp. 121-128. doi:10.1038/16393

[3] R. Saraswathi, M. Gerard and B. D. Malhotra, "Preparation and Measurements of Electrical and Spectroscopic Properties of Sodium Thiosulphate Doped Poly (o-toluidine)," Journal of Applied Polymer Science, Vol. 74, 1999, pp. 145-147.

doi:10.1002/(SICI)1097-4628(19991003)74:1<145::AIDAPP18>3.0.CO;2-C

[4] V. Ali, R. Kaur, N. Kamal, S. Singh, S. C. Jain, H. P. S. Kang, M. Zulfequar, M. M. Haq and M. Husain, "Synthesis and Characterization of Se Doped Poly (o-toluidine)," Journal of Physics Chemistry of Solids, Vol. 67, 2006, pp. 68-69.

[5] A. G. Macdiarmid, R. I. Mammone, J. R. Krawczyk and S. J. Porter, "Advanced Electronic and Photonic Materials and Devices," Molecular Crystals and Liquid Crystals, Vol. 105, 1984, pp. 89-105. doi: $10.1080 / 00268948408071645$

[6] A. G. MacDiarmid, J. C. Chiang, A. F. Rinchter, N. L. D. Somasiri and A. J. Epistein, "Synthesis and Characterization of Emeraldine Oxidation State by Elemental Analysis," Reidel Publisher, Dordrecht Holland, 1986.

[7] M. A. Majeed Khan, M. Zulfequar, A. Kumar and M. Husain, "Electrical Conductivity and Dielectric Properties of Sulfamic Acid Doped Poly(o-toluidine)," Materials Chemistry and Physics, Vol. 87, 2004, pp. 179-181.

[8] B. Scrosati, "Application of Electroactive Polymers," Chapman \& Hall, London, 1993. doi:10.1007/978-94-011-1568-1
[9] A. J. Epstein, J. Joo, R. S. Kohlman, G. Du, A. G. MacDiarmid, E. J. OH, Y. Min, J. Tsukamoto, H. Kaneko and J. P. Pouget, "Charge Transport Studies of Doped Poly (o-toluidine) with Various Dopants and Their Mixtures," Synthetic Metals, Vol. 65, No. 2-3, 1994, pp. 149-151. doi:10.1016/0379-6779(94)90176-7

[10] D. P. Gosain, T. Shimizu, M. Suzuki, T. Bando and S. Okano, "Optical Band Gap and Optical Constants in Amorphous Thin Films," Journal of Materials Science, Vol. 26, No. 12, 1991, pp. 3271-3273. doi:10.1007/BF01124673

[11] J. Fink and G. Leising, "Electronic Structure of Conducting Polymer: Investigations of Oriented Samples by Electron Energy Loss Spectroscopy," Physical Review B, Vol. 34, No. 1-3, 1986, pp. 5320-5322. doi:10.1103/PhysRevB.34.5320

[12] S. Kazim, V. Ali, M. Zulfequar, M. Haque and M. Husain, "Electrical, Thermal and Spectroscopic Studies of Te polyaniline," Current Applied Physics, Vol. 7, No. 1, 2006, pp. 68-75. doi:10.1016/j.cap.2005.11.072

[13] P. M. Grant and I. Batra, "Photoconductivity and Junction Properties of Polyacetylene Films," Solid State Communications, Vol. 29, 1979, pp. 225-227. doi:10.1016/0038-1098(79)91043-3

[14] J. Fink and G. Leising, "Momentum dependent Excitations in $\beta$-carotene a Finite-Size System between Molecules and Polymers," Physical Review B, Vol. 66, No. 15, 1991, pp. 2022-2025. doi:10.1103/physrevlett.66.2022

[15] S. Ameen, V. Ali, M. Zulfequar, M. M. Haq and M. Husain, "Preparation and Measurements of Electrical and Spectroscopic Properties of Sodium Thiosulphate Doped Polyaniline," Current Applied Physics, Vol. 9, No. 2, 2009. doi:10.1016/j.cap.2008.04.009 